\title{
Pattern search methods for pendant drops: Algorithms for rapid determination of surface tension and surfactant transport parameters
}

\author{
Andrew R. White, Thomas Ward* \\ Department of Aerospace Engineering, Iowa State University \\ Ames, IA 50011-2271
}

\begin{abstract}
In this manuscript we introduce algorithms based on the pattern search method that are used estimate equilibrium surface tension and surfactant transport parameters from pendant drops. The pattern search method is an efficient minimization technique for estimating multiple unknown parameters. To introduce and validate the method for measuring surface tension and transport parameters we use axisymmetric drop shape analysis (ADSA) for pendant drops of aqueous sodium oleate (SO) and aqueous sodium dodecyl sulfate (SDS) in mineral oil, along with several other classical data sets from the literature. The data show good agreement with other studies suggesting that the pattern search method may be a robust alternative to gradient based search methods.
\end{abstract}

* Corresponding author. Tel.: +1 515294 3935: fax: +1 5152943262

E-mail address: thomasw@iastate.edu (T. Ward). 


\section{Introduction}

In this manuscript we outline a process to apply pattern search methods to estimate equilibrium surface tension and surfactant transport parameters from pendant drops. The technique may be extended to other systems where robust minimization or search methods are required to estimate multiple unknown parameters. There are unique advantages with utilizing this technique to estimate properties of surfactant systems where minimization occurs between a known (experimental drop shapes or surface tension data) and unknown (Young-Laplace solution or isotherms) set of data, of which pendant drop analysis is an example. The main advantage stems from the fact that estimates for the unknown properties are produced by minimizing the common $\ell^{2}$-norm between the known and unknown data sets which can be used to formulate an objective function used for minimization. To validate the technique for measuring surface tension we consider pendant drops of aqueous sodium oleate (SO) and aqueous sodium dodecyl sulfate (SDS) in mineral oil, along with several other classical data sets from the literature. This will be followed by implementation of the pattern search method to fit the equilibrium surface tension data to the Frumkin isotherm which requires the simultaneous minimization of 3 unknown parameters used to quantify surfactant transport.

The analysis begins with estimates of surface tension data. To generate surface tension data an axisymmetric drop shape analysis (ADSA) for pendant drops will be considered. This generally consists of fitting drop shapes prescribed by the Young-Laplace equation to experimentally measured drop shapes. There are two unknown parameters in the ADSA process: 
the surface tension and the curvature. Constructing the objective function is the most robust method for determining the best fit using ADSA. But it is also the most costly since the numerically generated solution to the Young-Laplace equation requires solving differential equations in multiple dimensions. Furthermore, the range of surface tension and curvature values must be bounded in order to determine the region where the minimum exists along with choosing an appropriate size for incrementing the independent variables.

Within the past few decades gradient-based solvers have been developed to perform the ADSA minimization process. The most common example of such a method is through implementation of the well known Newton-Raphson scheme [1]. To perform the analysis the objective function is expanded in a Taylor series about the unknown parameters. Unfortunately gradient-based solvers too are computationally intensive, requiring the additional calculation of gradients to update the unknown in the iterative process. Additionally gradient-based solvers are not guaranteed to converge and tend to diverge if the initial guess is not sufficiently close to the best fit.

Instead we apply a pattern search method for determining best fits of the Young-Laplace equation. The pattern search method is an example of a direct search method which is more commonly used for performing modern error minimization. Direct search methods are less computational expensive than gradient based methods because they typically do not require any additional mathematical manipulation of an objective function. Drawbacks in utilizing these methods exist because they are not necessarily robust in terms of convergence to a 
local minimum. An example of a direct method is the Nelder-Mead simplex method [2, 3], generally recognized as the first non-gradient based search method. With this method, minimization is achieved by reaching the local minimum in a region usually defined by some $p+1$ points where $p$ is the number of unknowns, or dimensions. Points are updated by determining minima at points reflected through the line formed at the other $p$ points. This method has been used in [4] where the MATLAB function fminsearch was used to perform the implementation with good results. Although the Nelder-Mead simplex method is capable of producing good results there are no guarantees that it converges to a local minimum.

On the other hand the pattern search method has been shown through robust mathematical analysis to consistently converge to a local minimum the details of which were described in $[5,6]$. A brief analysis of why the pattern search method converges is as follows: an objective function based on the $\ell^{2}$-norm in multiple dimensions can possess a local minimum because the distance measured between the computational and experimental data $d_{i}$ is squared. For example, in one dimension let the distance between a point generated numerically by the Young-Laplace equation and one measured experimentally be a function of only the surface tension and be denoted $d_{i}\left(\gamma_{n}\right)$. Now bound $d_{i}\left(\gamma_{n}\right)$ above and below by adding and subtracting some small-equidistant amount $\delta$, respectively, from the unknown quantity $\gamma_{n}$. Then the error at step $n$ is bounded by $\gamma_{n} \pm \delta$ i.e. $\sqrt{d_{i}\left(\gamma_{n}\right)^{2}}<\sqrt{d_{i}\left(\gamma_{n} \pm \delta\right)^{2}}$. This condition forms the basis for the pattern search algorithm. We will explore how to implement this method, and discuss situations where the condition may break down in regards to determining equilibrium surface tension and surfactant transport parameters, in the following sections. 


\section{Pattern search algorithm}

\subsection{General pattern search algorithm}

We begin a discussion of the general pattern search algorithm that may be used to generate simultaneous estimates for multiple unknown parameters. The pattern search technique relies on the existence of a local minimum in an objective function for a set of unknown quantities. We will show that objective function estimates using an $\ell^{2}$-norm are sufficient to satisfy this criteria for determining equilibrium surface tension and surfactant transport parameters under certain conditions. The $\ell^{2}$-norm has been a standard for automated calculation of surface tension [1] and errors estimated using the $\ell^{2}$-norm can be generalized to any type of curve fitting method. The $\ell^{2}$-norm is simply defined by the Euclidean distance

$$
\psi\left(c_{1, n}, c_{2, n}, \ldots, c_{p, n}\right)=\sqrt{\sum_{i=1}^{I} d_{i}\left(c_{1, n}, c_{2, n}, \ldots, c_{p, n}\right)^{2}},
$$

where $\psi\left(c_{1, n}, c_{2, n}, \ldots, c_{p, n}\right)$ is the objective function of $p$ unknowns $c_{1, n}, c_{2, n}, \ldots, c_{p, n}$ which also serve to denote coordinates $\left(c_{1, n}, c_{2, n}, \ldots, c_{p, n}\right)$. The variable $d_{i}$ is used to denote the distance measured between $I$ points of some numerically generated and experimentally generated data sets at specific positions $i$ along an axis of the independent variable at minimization step $n$.

The general algorithm goes as follows: Starting with initial guesses for the coordinates $\left(c_{1,0}, c_{2,0}, \ldots, c_{p, 0}\right)$, we update these points to find a trajectory that leads to the local error 
minimum. For the pattern search algorithm this is achieved without the use of calculating gradients by determining the minimum in the set,

$$
\begin{aligned}
A=\quad & \left\{\psi\left(c_{1, n}+L_{1} \Delta c_{1, n}, c_{2, n}+L_{1} \Delta c_{2, n}, \ldots, c_{p, n}+L_{1} \Delta c_{p, n}\right),\right. \\
& \psi\left(c_{1, n}+L_{2} \Delta c_{1, n}, c_{2, n}+L_{1} \Delta c_{2, n}, \ldots, c_{p, n}+L_{1} \Delta c_{p, n}\right), \\
& \ldots \\
& \left.\psi\left(c_{1, n}+L_{3} \Delta c_{1, n}, c_{2, n}+L_{3} \Delta c_{2, n}, \ldots, c_{p, n}+L_{3} \Delta c_{p, n}\right)\right\}
\end{aligned}
$$

where $\left[L_{1}, L_{2}, L_{3}\right]=[-1,0,1]$. There are $3^{p}$ elements in set $A$ if one includes the initial coordinates at each minimization step $\left(c_{1, n}, c_{2, n}, \ldots, c_{p, n}\right)$. Let $\beta_{1}, \beta_{2}, \ldots, \beta_{p}=1,2$ or 3 be used to denote the indices $L_{\beta_{1}}, L_{\beta_{2}}, \ldots, L_{\beta_{p}}$ corresponding to the objective function minimum at step $n$ in set $A$ located at coordinates $\left(c_{1, n}+L_{\beta_{1}} \Delta c_{1, n}, c_{2, n}+L_{\beta_{2}} \Delta c_{2, n}, \ldots, c_{p, n}+L_{\beta_{p}} \Delta c_{p, n}\right)$. If the elements of the vector $\mathbf{M}=\left(M_{1}, M_{2}, \ldots, M_{p}\right)=\left(L_{\beta_{1}}, L_{\beta_{2}}, \ldots, L_{\beta_{p}}\right)$ contain these values then new guesses at step $n+1$ for the unknowns can be written as

$$
\left[\begin{array}{c}
c_{1, n+1} \\
c_{2, n+1} \\
\ldots \\
c_{p, n+1}
\end{array}\right]=\left[\begin{array}{c}
c_{1, n} \\
c_{2, n} \\
\cdots \\
c_{p, n}
\end{array}\right]+\left[\begin{array}{c}
M_{1} \Delta c_{1, n+1} \\
M_{2} \Delta c_{2, n+1} \\
\cdots \\
M_{p} \Delta c_{p, n+1}
\end{array}\right] .
$$

The step sizes $\Delta c_{1, n+1}, \Delta c_{2, n+1}, \ldots \Delta c_{p, n+1}$ remain constant until the minimum of set $A$ produces the zero vector i.e. $\beta_{1}=\beta_{2}=\cdots=\beta_{p}=2$ such that $\mathbf{M}=\mathbf{0}$. If this occurs then the step size is uniformly reduced by $\phi$ i.e. $\Delta c_{1, n+1}, \Delta c_{2, n+1}, \ldots \Delta c_{p, n+1}=$ 
$\phi \Delta c_{1, n+1}, \phi \Delta c_{2, n+1}, \ldots \phi \Delta c_{p, n+1}$ with $0<\phi<1$ and the procedure continues. The iterative process is completed when one or several of the unknowns meet a user defined minimum error requirement between two consecutive steps $\left|c_{1, n+1}-c_{1, n}\right|$ or $\left|c_{2, n+1}-c_{2, n}\right|$ or $\ldots$ or $\mid c_{p, n+1}-$ $c_{p, n} \mid \ll 1$.

\subsection{Log scaling}

In situations where one or more of the $p$ unknowns contains values that can span multiple orders of magnitude it is possible to use a log scale. For any unknowns to be treated in this manner its coordinates for $\psi$ in $A$ would be written as,

$$
\begin{aligned}
A= & \left\{\psi\left(c_{1, n} 10^{L_{1} \Delta c_{1, n}}, c_{2, n} 10^{L_{1} \Delta c_{p, n}}, \ldots, c_{p, n} 10^{L_{1} \Delta c_{p, n}}\right),\right. \\
& \psi\left(c_{1, n} 10^{L_{2} \Delta c_{1, n}}, c_{2, n} 10^{L_{1} \Delta c_{p, n}}, \ldots, c_{p, n} 10^{L_{1} \Delta c_{p, n}}\right), \\
& \ldots \\
& \left.\psi\left(c_{1, n} 10^{L_{3} \Delta c_{1, n}}, c_{2, n} 10^{L_{3} \Delta c_{p, n}}, \ldots, c_{p, n} 10^{L_{3} \Delta c_{p, n}}\right)\right\} .
\end{aligned}
$$

A base of 10 is used for this example but any value can be used with the algorithm. The rest of the algorithm is the same with the exception being the new guesses are written:

$$
\left[\begin{array}{c}
c_{1, n+1} \\
c_{2, n+1} \\
\cdots \\
c_{p, n+1}
\end{array}\right]=\left[\begin{array}{c}
c_{1, n} 10^{M_{1} \Delta c_{1, n+1}} \\
c_{2, n} 10^{M_{2} \Delta c_{2, n+1}} \\
\cdots \\
c_{p, n} 10^{M_{p} \Delta c_{p, n+1}}
\end{array}\right]
$$


The iterative process is complete when the error is minimized based on user defined values.

\subsection{Implementation}

Implementation requires determining the set of values at each set of coordinates that are included in $A$. Any looping algorithm will produce the desired set of values. What is important is how the coordinates are addressed. For this step in the algorithm it is convenient to think of the coordinates as forming a stencil with a central point being the initial coordinate for the current minimization step $\left(c_{1, n}, c_{2, n}, \ldots, c_{p, n}\right)$. Using the central point as the initial point in coordinate addressing is not recommended since it will be inefficient when programming.

Instead the $\left(c_{1, n}+L_{1} \Delta c_{1, n}, c_{2, n}+L_{1} \Delta c_{2, n}, \ldots, c_{p, n}+L_{1} \Delta c_{p, n}\right)\left(L_{1}=-1\right)$ coordinate should be addressed as the 1 st point. Then the central point will be the 5 th point if numbering from left to right followed by top to bottom in two dimensions (two unknowns) for example.

Central to building an efficient algorithm is developing a process for determining the vector M. In practice the vector $\mathbf{M}$ does not need to be determined explicitly. Instead the new points may be updated using the addressing described above. Then by pre-assigning $L_{1}, L_{2}$ and $L_{3}$ values to the addresses in the stencil it is possible to build a fast algorithm without the need to explicitly determine the vector $\mathbf{M}$. Referring back to the two dimensional example if the address one is the minimum for the current step then this would correspond to $L_{\beta_{1}}=L_{\beta_{2}}=-1$ or the upper left corner of the stencil. 
The details above provide a formal mathematical statement for implementing the pattern search algorithm. In practice the implementation is rather straightforward and intuitive as we discuss in the following sections using pendant drop analysis and estimates for surfactant transport parameters as examples.

\section{Pendant drops}

\subsection{Image analysis and fitting procedure}

The first step in ADSA is accurately defining the experimental drop surface. This step alone has warranted a detailed analysis from other researchers [7]. For this manuscript we use a pixel threshold analysis [8] to define pixels occupied by the continuous or drop phases. Then, beginning with the top left edge of the drop where it meets the needle, the drop surface is traced using the following algorithm. Consider the schematic of the drop surface shown in Fig. 1(a) following the application of the pixel threshold where dark squares are pixels occupied by the drop, gray squares are occupied by the continuous phase and squares outlined in white represent the drop surface. The pixel ' $\mathrm{X}$ ' is known to be on the surface. Its eight neighboring pixels are considered in counterclockwise order beginning with the pixel to the right. Once a dark (drop-containing) pixel is found that pixel is saved as another point on the surface. Next at the newly discovered surface point, its eight neighboring pixels are now checked in counterclockwise order. A crucial rule in this algorithm is the first pixel that is checked of the eight neighboring pixels is the last non-surface pixel checked from the previous set of eight. The arrows in Fig. 1 show which pixels are checked and in what order as the 
a)

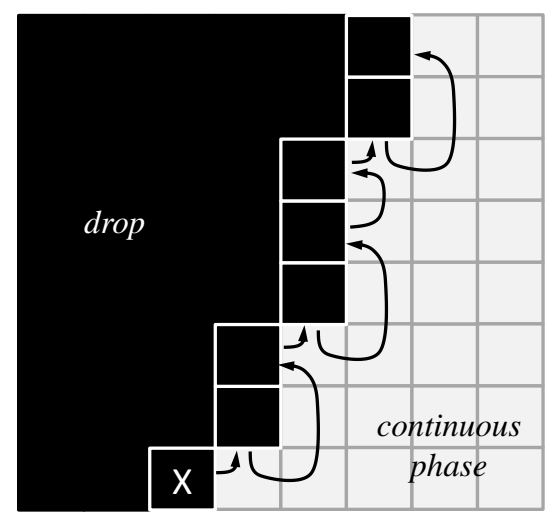

b)

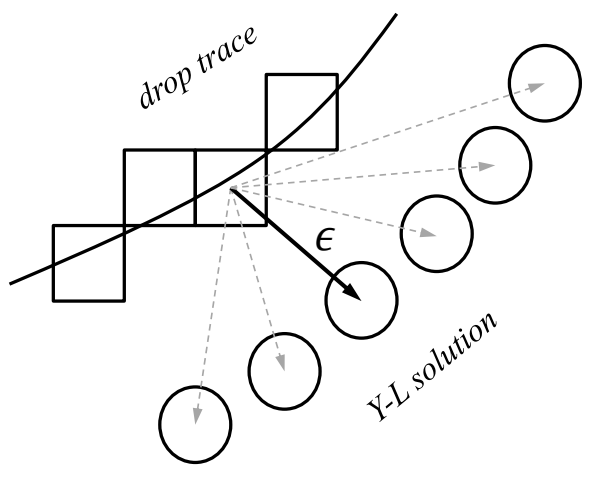

Figure 1: in (a) a visual representation of the tracing algorithm is shown. In this schematic the algorithm begins at ' $\mathrm{X}$ ' and continues in the path indicated by the arrows. In (b) a visual representation of the method used to determine $\epsilon$ and subsequently $\psi(b, \gamma)$ is shown where squares represent coordinates of $I=\left(x_{i}, z_{i}\right)$ and circles represent coordinates of $Y L=$ $\left(x_{j}, z_{j}\right)$.

surface is defined. This algorithm continues until the right edge of the needle tip is reached.

The tracing step results in a set of interface coordinates, denoted $I=\left(x_{i}, z_{i}\right)$. Next the experimental drop shape is compared to a drop shape prescribed by the Young-Laplace equation, $\gamma_{n} \kappa=\Delta \rho g z-\frac{2 \gamma}{b_{n}}$, where $\kappa$ is the surface curvature, $\gamma_{n}$ is the unknown surface tension and $b_{n}$ is the unknown radius at the drop apex. Rearranging and non-dimensionalizing the Young-Laplace equation using the unknown $b_{n}$ as the length scale yields $\kappa^{*}=B o^{*} z^{*}-2$ where $B o^{*}=\Delta \rho g b_{n}^{2} / \gamma_{n}$ is the Bond number. The superscript ${ }^{*}$, indicates dimensionless quantities. In order to determine a drop's shape from this equation it is parameterized with 
respect to arc length $s$, yielding

$$
\begin{gathered}
\frac{d \theta}{d s^{*}}=2-B o^{*} z^{*}-\frac{\sin \theta}{x^{*}} \\
\frac{d x^{*}}{d s^{*}}=\cos \theta \\
\frac{d z^{*}}{d s^{*}}=\sin \theta
\end{gathered}
$$

where $\theta$ is the angle between the surface tangent and the axis of symmetry. For a derivation of this set of differential equations readers should refer to $[1,9,10]$. An adaptive 4 th-order explicit Runge-Kutta-Merson scheme is used to numerically integrate these equations for a given $B o^{*}$ with appropriate initial conditions. The solution is initialized at $\theta \rightarrow 0$ where $z^{\prime} \rightarrow 0$ (primes indicate derivatives with respect to $s$ ) and the Young-Laplace equation can be approximated by the ordinary differential equation $z^{\prime \prime}+z^{\prime} / x+B o^{*} z^{*}-2=0$. This has the analytical solution

$$
z^{*}=\frac{2}{B o^{*}}\left[1-J_{0}\left(x^{*} \sqrt{B o^{*}}\right)\right]
$$

where $J_{0}$ is the zeroth order Bessel function of the first kind $[9,11]$. The drop shape given by eqs. 4-6 is denoted $Y L=\left(x_{j}, z_{j}\right)$.

Solutions of the Young-Laplace equation presented above are symmetric about the apex, so $I$ is split into two halves about the vertical axis of symmetry. The horizontal location of the axis of symmetry $x_{s}$ is measured to be the average of the leftmost and rightmost horizontal 

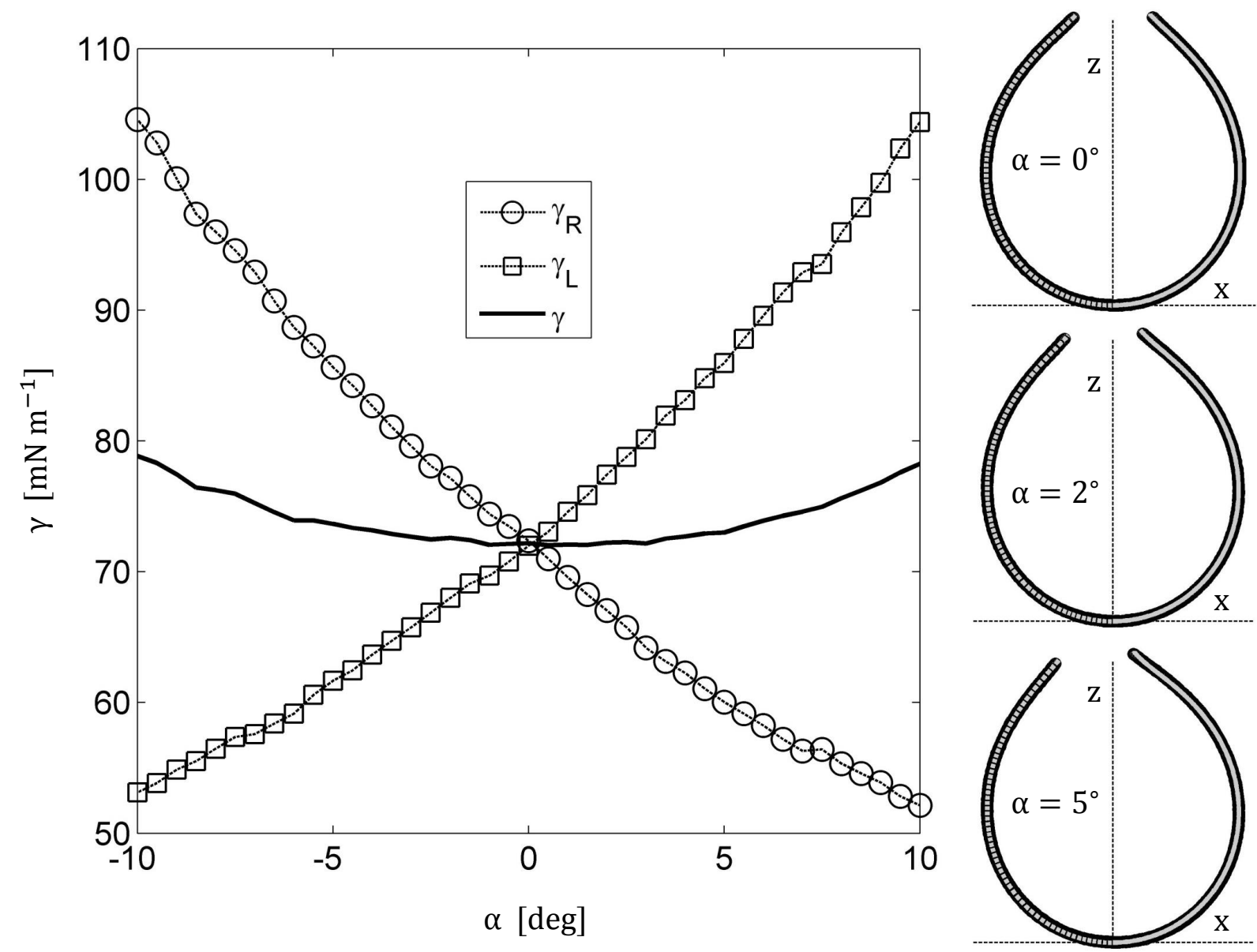

Figure 2: the effect of drop tilt on $\gamma$ is shown for the experimental trace $I$ of a $7.75 \mu$ drop of water suspended in air. The trace is rotated $\pm 10^{\circ}$. Three examples of $I$ (black) with the best fits $\mathrm{YL}_{R}$ (solid gray) and $\mathrm{YL}_{L}$ (dotted gray) are shown for $0^{\circ}, 2^{\circ}$ and $5^{\circ}$.

coordinates of $I$. The resulting left and right halves are $I_{L}=\left(x_{\ell}, z_{\ell}\right)$ and $I_{R}=\left(x_{r}, z_{r}\right)$ which contain $L$ and $R$ coordinate points, respectively. The maximum horizontal distance between the axis of symmetry and the drop surface is denoted $b_{x}$ and is the initial guess for the radius of curvature at the drop apex, $b_{0}$.

For every point on the interface the minimum Euclidian distance between the experimen- 
tal and Young-Laplace drop shapes is needed. This is defined by the minimum of $d_{i}=$ $\sqrt{\left(x_{i}-x_{j}\right)^{2}+\left(z_{i}-z_{j}\right)^{2}}$ where $\left(x_{j}, z_{j}\right)$ are coordinates of $Y L$ and $\left(x_{i}, z_{i}\right)$ are coordinates of $I_{R}$ or $I_{L}$. This distance is denoted $\epsilon$ and is shown schematically in Fig. 1(b). The sum of all $\epsilon$ normalized by $L$ or $R$ for $I_{L}$ or $I_{R}$, respectively, gives the objective functions $\psi_{L}\left(b_{n}, \gamma_{n}\right)$ or $\psi_{R}\left(b_{n}, \gamma_{n}\right)$ for a particular guess of $\gamma_{n}$ and $b_{n}$. There are $p=2$ unknown parameters, $\left(c_{1, n}, c_{2, n}\right)=\left(b_{n}, \gamma_{n}\right)$, for the pattern search method applied to pendant drops. Therefore, the set $A$ from eq. 2 will contain $3^{p}$ points producing a nine-point stencil of values.

As stated above the initial guess for $b_{n}$ is $b_{x}$. To determine an initial $\gamma_{n}$ an initial guess of $B o^{*} \approx 0.2$ is made such that $\gamma_{0}=5 \Delta \rho g b_{x}^{2}$. Depending on the surfactant system, $\gamma_{n}$ can take values from approximately $O(10)$ to $O(0.0001) \mathrm{mN} \mathrm{m}^{-1}$. For this reason a log scale is used for the stencil in the $\gamma$-direction in the form $\gamma_{n} 10^{L \Delta \gamma^{*}}$ with $\Delta \gamma_{n}^{*}=0.05$ initially and $L=[-1,0,1]$. For the $b_{n}$-direction the stencil is scaled linearly like $b_{n}+L \Delta b_{n}$ with $\Delta b_{n}$ being $10 \mu \mathrm{m}$ initially, and $\phi$ is fixed to $\phi=0.1$. The pattern search algorithm continues until $\left(\gamma_{n} 10^{\Delta \gamma_{n}^{*}}-\gamma_{n}\right) / \gamma_{n}<10^{-4}$ and $\Delta b_{n} / b_{n}<10^{-4}$. To ensure that the global minimum has been found, the algorithm is restarted using the previously converged $b_{n}$ and $\gamma_{n}$ as the initial guess. If the $b_{n}$ and $\gamma_{n}$ from successive runs of the pattern search algorithm are sufficiently close then those $b_{n}$ and $\gamma_{n}$ are taken as the solution.

This process is carried out to fit $Y L$ to both $I_{L}$ and $I_{R}$, giving two estimates for surface tension, $\gamma_{L}$ and $\gamma_{R}$, for the drop. The average of the values for the two halves then gives $\gamma$ for the drop at that instant in time. It is unnecessary to add a third unknown, the possible 
angle of the drop with respect to gravity due to camera misalignment $\alpha$, if $\gamma$ is determined with this algorithm. Consider Fig. 2 where the experimental trace $I$ of the pendant drop in Fig. 4(a) has been artificially rotated $\alpha= \pm 10^{\circ}$. Values for $\gamma_{R}$ and $\gamma_{L}$ deviate significantly as $|\alpha|>0^{\circ}$, but the mean $\gamma$ over $-2^{\circ} \leq \alpha \leq 2^{\circ}$ is $72.13 \mathrm{mN} \mathrm{m}^{-1}$ with a standard deviation of $0.09 \mathrm{mN} \mathrm{m}^{-1}$ or $0.12 \%$ of the mean. Over $-1^{\circ} \leq \alpha \leq 1^{\circ}$ the mean $\gamma$ becomes $72.12 \mathrm{mN}$ $\mathrm{m}^{-1}$ with a standard deviation of $0.06 \mathrm{mN} \mathrm{m}^{-1}$ or $0.08 \%$ of the mean. For $|\alpha|>2^{\circ}$ then the errors in $\gamma$ become significant. However misalignment angles between 1 and $2^{\circ}$ and larger are noticeable with the naked eye as demonstrated in the example fits on the right side of Fig. 2. Misalignment angles at these values were therefore corrected prior to image capture, leaving $\alpha$ small enough that any potential errors would be around $0.1 \%$ or less. Finally, in the case of fitting successive images the initial guesses $b_{0}$ and $\gamma_{0}$ for the next image are taken as the solution of the previously fit image.

\subsection{Experiments}

An in-house setup was created to image pendant drops and bubbles for analysis similar to many common tensiometers. A vertically oriented syringe pump (New Era Pump Systems) drove the plunger of a $100 \mu$ l Hamilton Gastight syringe. The syringe needle was a 22 gauge stainless steel type 3 needle (Hamilton) for pendant drops and either a 22 or 16 gauge hooked "J-needle" (Ramé-Hart) for pendant bubbles. A clear $25 \times 25 \times 50 \mathrm{~mm}$ acrylic box

was used to contain the continuous phase. A CCD camera (PixeLINK) captured images of the drop or bubble with a resolution of $1200 \times 1600$ pixels. The aspect ratio of the images was calibrated by orienting a standard 22 gauge syringe needle both vertically and horizon- 
tally, checking for differences in observed needle thickness of which none was found. A 15 W vertical fluorescent lamp was situated on the side of the drop or bubble opposite the camera. This provided excellent contrast between the drop or bubble phase and the continuous phase.

The surfactants used in pendant drop experiments were either $>99 \%$ sodium dodecyl sulfate (SDS) (Fisher Scientific) or $>97 \%$ sodium oleate $(\mathrm{SO})$ (TCI). Both surfactants were used as

received. SDS concentrations ranged between 0.1 to $5.0 \mathrm{mM}$ and $\mathrm{SO}$ concentrations ranged between 0.04 to $2.0 \mathrm{mM}$. The continuous phases were either air, light mineral oil (Fisher Scientific, $\rho_{d}=830 \mathrm{~kg} \mathrm{~m}^{-3}$ ) or reverse osmosis water at $23^{\circ} \mathrm{C}$. Drop volumes ranged from 1 to $30 \mu \mathrm{l}$ so that the drops were both not too spherical and remained attached to the needle.

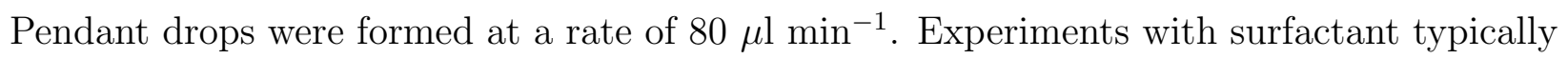
lasted between 5 and 30 minutes. Images were taken of the drops over time at intervals of 2 to $30 \mathrm{~s}$ per frame and were processed in MATLAB as described in the previous section.

\subsection{Results: Pendant drops}

Figure 3 shows example contour plots of $\psi_{R}(b, \gamma)$ and the subsequent path generated by minimizing the objective function using the pattern search algorithm for the drop's right halves $\left(I_{R}\right)$. Four pendant drop/bubble systems are considered: two drops of water with different volumes suspended in air, an air bubble in water, and a water drop with $5.6 \mathrm{mM}$ SO suspended in corn oil (Crisco) for 10 minutes. The contour plots of the objective functions were generated by solving eqs. 4-6 for the range of $b$ and $\gamma$ shown. In each subplot the 

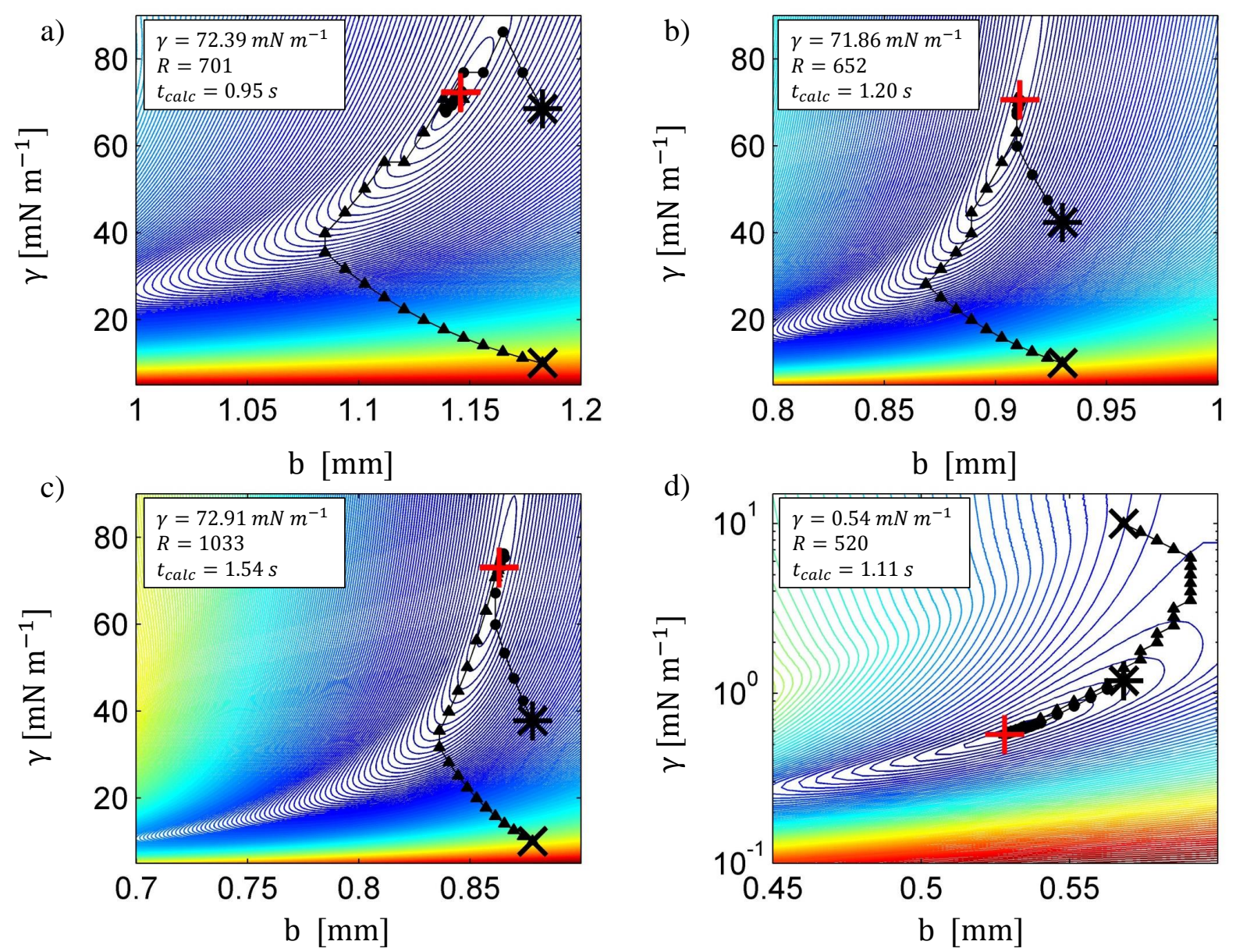

Figure 3: example contour plots of $\psi(b, \gamma)$ are shown for (a) a $7.75 \mu$ l water drop in air, (b) a $3.5 \mu \mathrm{l}$ water drop in air, (c) a $3 \mu \mathrm{l}$ air bubble in water, and (d) a $0.9 \mu \mathrm{l} 5.6 \mathrm{mM}$ SO drop in corn oil after 10 minutes. The '*' indicates the initial guess using $\gamma=5 \Delta \rho g b_{x}^{2}$ and the 'X' indicates another run of the algorithm starting at $\gamma=10 \mathrm{mN} \mathrm{m}^{-1}$. The red ' + ' indicates the best fit while the black circles and triangles indicate successive guesses made by the pattern search algorithm. The resulting surface tension values are annotated in the top left of each plot along with the number of points on the right interface $R$ and the computational time $t_{\text {calc }}$ in seconds. 
${ }^{\prime}{ }^{\prime}$ indicates the initial guess using $\gamma_{0}=5 \Delta \rho g b_{x}^{2}$ with the black circles being the successive guesses by the pattern search algorithm. The ' $X^{\prime}$ indicates another run of the algorithm with an initial guess of $10 \mathrm{mN} \mathrm{m}^{-1}$ and the black triangles are its successive guesses. The red ' + ' indicates the best fit. With the exception of Fig. 3(d) the axes of each figure are scaled identically. In the top left of each plot in Fig. 3 the best fit surface tension is shown along with the number of points $R$ in $I_{R}$. The computation time $t_{c a l c}$ required to reach $\gamma_{R}$ when using the pattern search algorithm with the initial guess of $\gamma_{0}=5 \Delta \rho g b_{x}^{2}$ using a computer with a $3.60 \mathrm{GHz}$ Intel $\mathrm{CPU}$ is also shown.

Figure 4 shows raw experimental images with best fits of eqs. 4-6 in white corresponding to the contour plots in Fig. 3. The volume and $B o^{*}$ for each drop is shown. The values for $\gamma_{R}$ and $\gamma_{L}$ for the drop in Fig. 3(a) are 72.39 and $72.01 \mathrm{mN} \mathrm{m}^{-1}$, respectively, resulting in $\gamma=72.20 \mathrm{mN} \mathrm{m}^{-1}$ which agrees well with values in the literature [12].

\section{Surfactant transport parameters}

\subsection{Isotherm and equation of state analysis}

After determining equilibrium values for the surface tension $\gamma_{e q}$ it is common to further manipulate that data to estimate surfactant transport parameters for the surfactant-fluid systems $[13,14]$. Expressions governing surfactant sorption along and interface are typically written in terms of the local surface coverage of surfactant $\Gamma_{e q}\left(\mathrm{~mol} \mathrm{~m}^{-2}\right)$ as a function 
a)

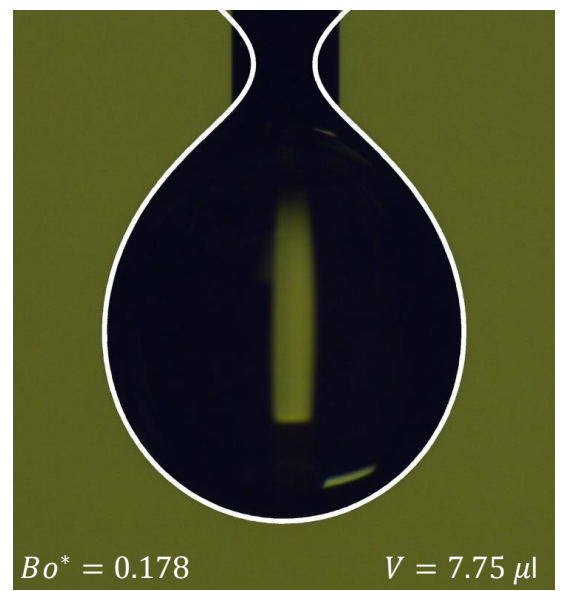

c)

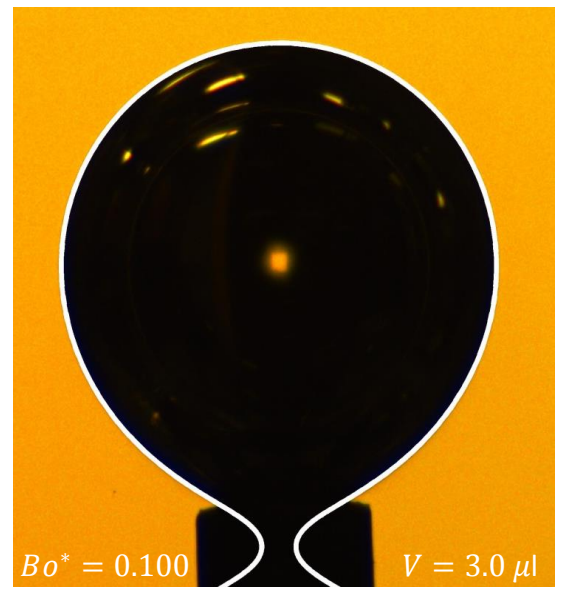

b)

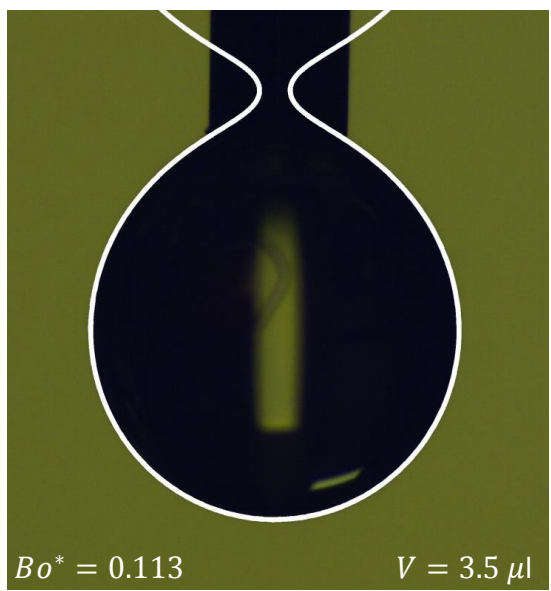

d)

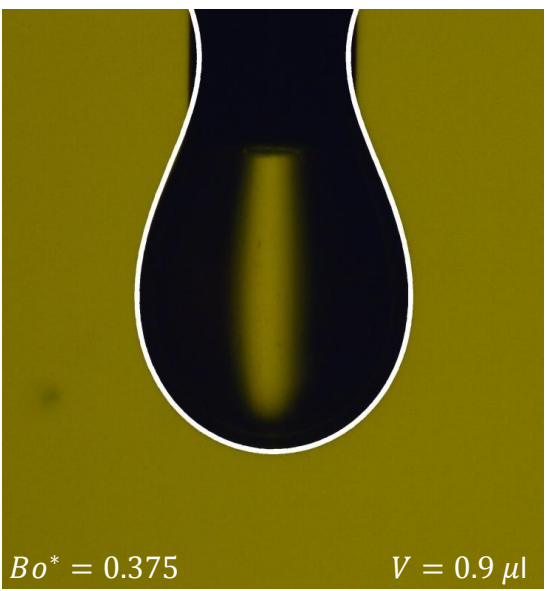

Figure 4: pendant drops or bubbles corresponding to the contour plots of $\psi(b, \gamma)$ in Fig. 3 are shown for (a) a $7.75 \mu$ l water drop in air, (b) a $3.5 \mu$ l water drop in air, (c) a $3 \mu \mathrm{l}$ air bubble in water, and (d) a $0.9 \mu \mathrm{l} 5.6 \mathrm{mM}$ SO drop in corn oil after 10 minutes. The white line is the best fit of eqs. 4-6. The Bond numbers are annotated for each drop or bubble. 
of the subsurface concentration $C_{s}\left(\mathrm{~mol} \mathrm{~m}^{-3}\right)$. Units for each of the quantities discussed in this section are provided to help avoid confusion with other studies that utilize similar notation. The Frumkin isotherm is one of the most commonly used expressions for measuring or estimating surfactant transport parameters [15]. It relates transport properties such as the bulk surfactant distribution $K_{e q}\left(\mathrm{~m}^{3} \mathrm{~mol}^{-1}\right)$, maximum surface coverage $\Gamma_{\infty}\left(\mathrm{mol} \mathrm{m}{ }^{-2}\right)$ at or above the critical micellar concentration $C_{\infty}$, and a molecular interaction parameter, $\lambda:$

$$
\frac{\Gamma_{e q}}{\Gamma_{\infty}}=\frac{K_{e q} C_{s}}{e^{-\lambda \Gamma_{e q} / \Gamma_{\infty}}+K_{e q} C_{s}}
$$

We note that use of the Gibbs adsorption equation, $d \gamma_{e q}=-R T \Gamma_{e q} d \ln C$, with the Frumkin isotherm yield the familiar equation of state,

$$
\gamma_{e q}=\gamma_{0}+m R T \Gamma_{\infty}\left[\ln \left(1-\frac{\Gamma_{e q}}{\Gamma_{\infty}}\right)+\frac{1}{2} \lambda\left(\frac{\Gamma_{e q}}{\Gamma_{\infty}}\right)^{2}\right]
$$

Here $\gamma_{0}$ is the surface tension for $\Gamma_{e q}=0, R\left(\mathrm{~J} \mathrm{~mol}^{-1} \mathrm{~K}^{-1}\right)$ is the universal gas constant and $T(\mathrm{~K})$ the temperature. The constant $m$ changes value depending on the surfactant where non-ionic surfactants have a value of $m=1$ while $1: 1$ ionic surfactants require a value of $m=2$ to account for the species generated by dissociation $[16,17,18,19]$. Using these two equations it is possible to predict how $\gamma_{e q}$ varies with $C_{s}$ for given $K_{e q}, \Gamma_{\infty}$ and $\lambda$. Thus $K$, $\Gamma_{\infty}$ and $\lambda$ can be chosen such that the norm between the resulting $\gamma_{e q}$ versus $C$ curve and the curve generated from experiments is minimized using a pattern search algorithm. 
For $\lambda=0$ the system reduces to the Langmuir isotherm where convergence is guaranteed. The Frumkin isotherm with $\lambda \neq 0$ can present several challenges for convergence when using the pattern search algorithm. First, consider that the isotherm and equation of state are difficult to manipulate because the system of equations actually has four unknowns, $\Gamma_{e q}, \Gamma_{\infty}$, $K_{e q}$ and $\lambda$, with only two equations used to determine them. Here we have generalized the system of equations by considering $\Gamma_{e q}$ as an unknown since its value depends on $\Gamma_{\infty}$ and $\lambda$ according to the equation of state. Furthermore, the value of $\lambda$ is bounded above by 4 as discussed in [15]. Values $\lambda>4$ yield isotherms in the $\Gamma_{e q}-C_{s}$ plane that possess multiple $\Gamma_{e q}$ values at a given concentration.

Convergence is also directly influenced by the molecular interaction parameter for $\lambda \leq 4$. To introduce a convvergence criteria for the pattern search method we determine a mathematical relationship between the maximum surface coverage $\Gamma_{\infty}$ and $\lambda$ using the Frumkin isotherm. The criteria is developed by expanding the exponential term in the denominator of eq. 8 for small $\lambda \Gamma_{e q} / \Gamma_{\infty}$ yielding the linearized Frumkin isotherm equation $\Gamma_{e q} / \Gamma_{\infty}=K_{e q} C_{s} /[1-$ $\left.\lambda \Gamma_{e q} / \Gamma_{\infty}+K_{e q} C_{s}\right]$ where we have dropped the $\mathrm{O}\left(\lambda \Gamma_{e q} / \Gamma_{\infty}\right)^{2} \ldots$ terms from the expansion. The maximum surface coverage occurs at or above the critical micellar concentration so we set $C_{s}=C_{\infty}$ in the resulting expression. Collecting terms involving $\Gamma_{\infty}$ we derive the quadratic equation:

$$
\frac{\Gamma_{\infty}}{\Gamma_{e q}}=\frac{1+K_{e q} C_{\infty}}{2 K_{e q} C_{\infty}} \pm \sqrt{\left[\frac{1+K_{e q} C_{\infty}}{4 K_{e q} C_{\infty}}\right]^{2}-\frac{\lambda}{K_{e q} C_{\infty}}}
$$


for the limit of small $\lambda \Gamma_{e q} / \Gamma_{\infty}$. For $\lambda=0$ there is a unique non-trivial solution to this equation, meaning that the Langmuir isotherm will absolutely converge to the correct $\Gamma_{\infty}$ and $K_{e q}$. In terms of understanding the influence of $\lambda \neq 0$ on convergence notice that positive $\lambda<4$ can produce a discriminant with imaginary values provided $\lambda>\lambda_{\text {crit }}$ where $\lambda_{\text {crit }}=\left[1+K_{e q} C_{\infty}\right]^{2} /\left[4 K_{e q} C_{\infty}\right]$. In terms of curve fitting using the pattern search algorithm this is ideal since only real values would be inputs or outputs i.e. there is a unique real solution for $\Gamma_{\infty} / \Gamma_{e q}$. This would suggest that systems possessing large positive values for $4>\lambda>\lambda_{\text {crit }}$ will conditionally converge to the correct values for $\Gamma_{\infty}$ and subsequently

$K_{e q}$. But for $\lambda<\lambda_{\text {crit }}$ the discriminant is real suggesting that it is possible to have two real solutions for $\Gamma_{\infty} / \Gamma_{e q}$. Therefore, any negative $\lambda$ satisfies this condition since $K_{e q}>0$ and $C_{\infty}>0$. This is a problematic condition for any minimization algorithm because it suggests two minima exists. Furthermore, if $\lambda$ is not close to zero then the expansion of the exponential term performed above requires higher order polynomials in $\Gamma_{\infty}$ for better accuracy. So there is a potential for producing multiple local minima when using the pattern search algorithm if $\lambda<0$.

\subsection{Results: Sorption kinetics}

While the convergence of the objective function for the isotherm and equation of state is conditional the equations themselves are straightforward to manipulate and do not require integration. For this discussion we will focus on using the unknown parameters $\left(c_{1, n}, c_{2, n}, c_{3, n}\right)=\left(K_{n}, \Gamma_{\infty n}, \lambda_{n}\right)$ in eq. 8 to develop a pattern search algorithm. One only needs to provide $\left(K_{e q, 0}, \Gamma_{\infty, 0}, \lambda_{0}\right)$ for a range of bulk concentrations $C$ where $C_{s}=C$ when 
neglecting depletion. Then eq. 8 must be iterated to find $\Gamma_{e q}$ for that range of concentrations. For this process we use a bisect method with tolerance control to iterate the solution until the errors between two consecutive steps is less than $5 \times 10^{-16}$. Once these values are found the $\Gamma_{e q}$ values are input into eq. 9 and the norm can be determined as the difference between the experimental and numerical values for the surface tension denoted $\gamma_{e q, e x p}$ and $\gamma_{e q, \text { num }}$, respectively.

Initial values for the unknowns $\left(c_{1,0}, c_{2,0}, c_{3,0}\right)=\left(K_{e q, 0}, \Gamma_{\infty, 0}, \lambda_{0}\right)$ are chosen based on available data for these parameters. The bulk surfactant distribution coefficient $K_{e q, 0}$ is never negative so we choose values that are initially greater than 1 . The $\Delta K_{e q}$ is chosen to be $0.1 \Delta K_{e q}$. The molecular interaction parameter $\lambda_{0}$ must be less than 4 , and can be negative. To remove any bias from the initial choice we choose this value as initially zero with $\Delta \lambda=0.1$. The maximum surface coverage $\Gamma_{\infty}$ can have the most salient influence on the final result because, as mentioned in previous paragraphs, $\Gamma_{e q}$ is also an unknown and the ratio of these two variables appears in both the isotherm and equation of state. We choose an initial value of $\Gamma_{\infty, 0}=1 \times 10^{-5}$. The value for $\Delta \Gamma_{\infty}=0.01 \Gamma_{\infty, 0}$ is seen to provide fast convergence. Note that the stencil size for this algorithm is $3^{p}$ where $p=3$ or 27 . So 27 calculations are required to determine the path to the local minimum if it exists. Assigning address values begin from left to right, top to bottom, then front to back. Therefore, the central coordinate, used as the location for the initial and updated point, is located at address 14.

The algorithm is applied to several sets of data for $\gamma$ versus $C$ to show the range results when 

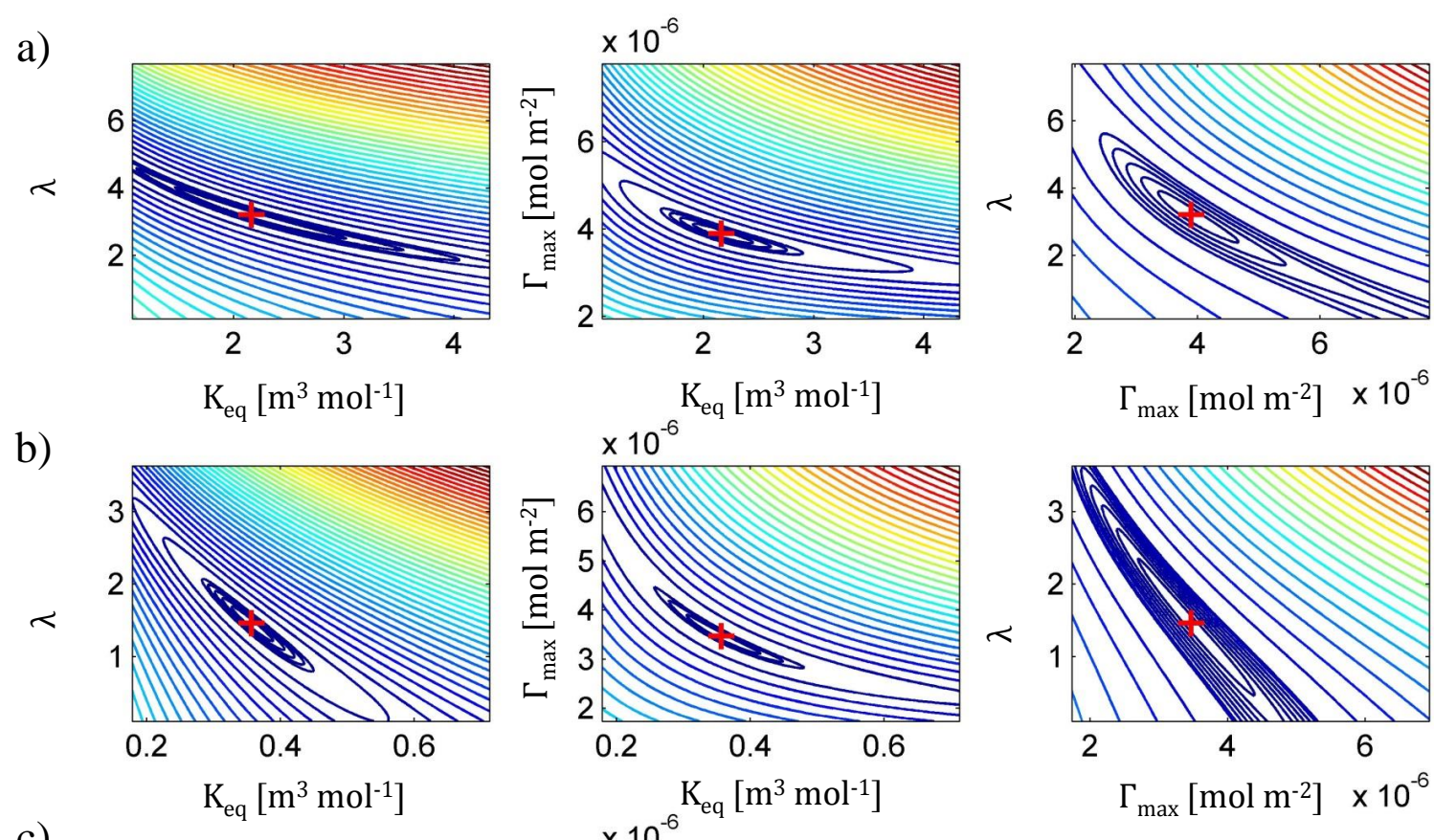

c)
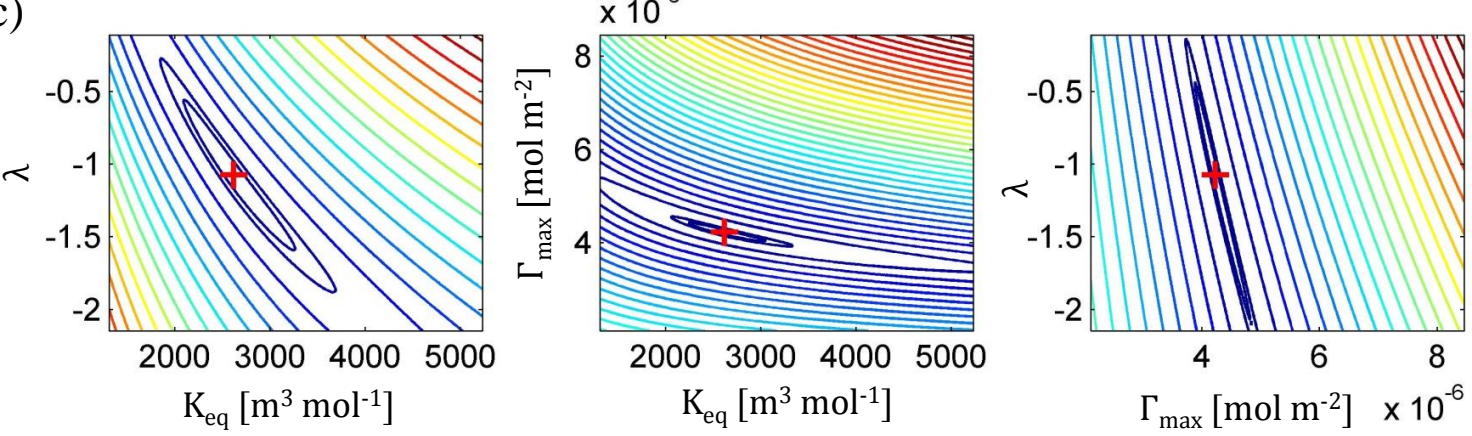

Figure 5: Graphs of the objective function in either $\lambda-K_{e q}, \Gamma_{\infty}-K_{e q}$ or $\lambda-\Gamma_{\infty}$ plane for a) aqueous SO in mineral oil (pendant drop), b) SDS in air [23] (du Noüy ring) and c) aqueous $\mathrm{C}_{12} \mathrm{E}_{4}$ in air (pendant drop) [19]. The red + shows the equilibrium value found using the pattern search algorithm. 

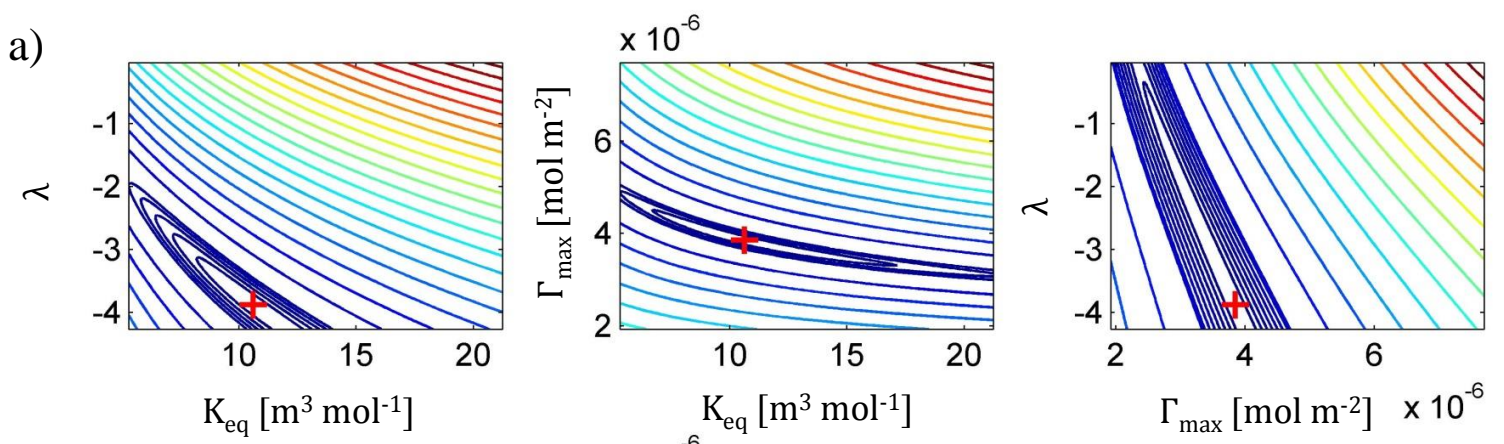

b)
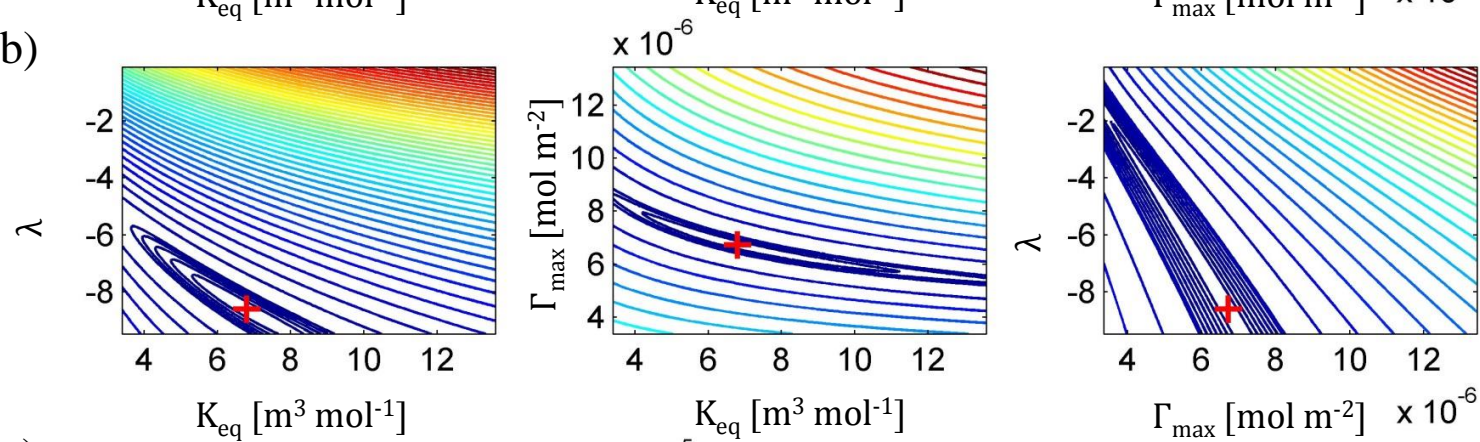

c)
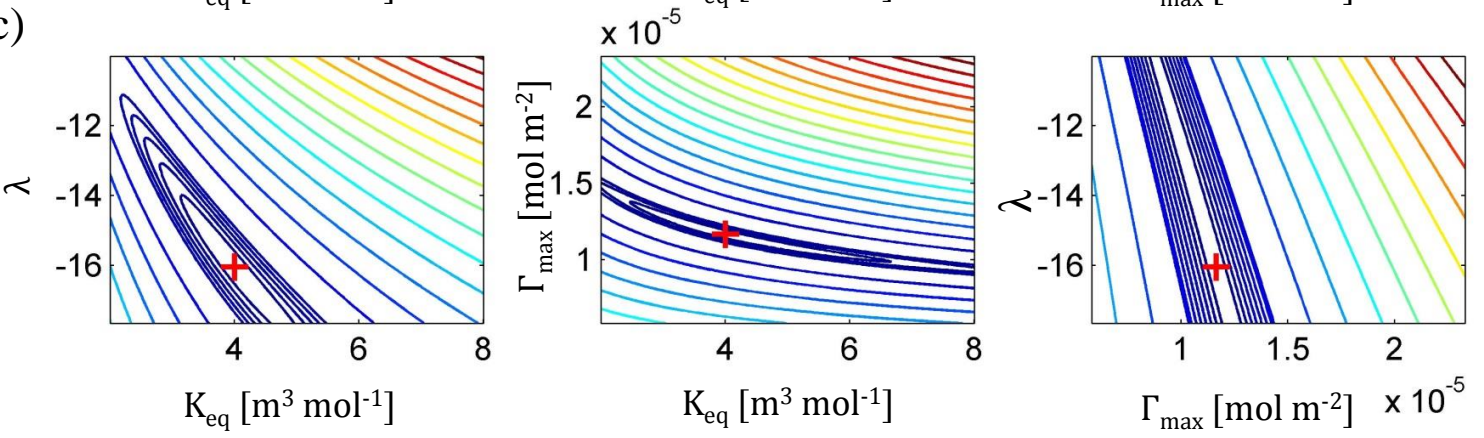

Figure 6: Graphs of the objective function in either $\lambda-K_{e q}, \Gamma_{\infty}-K_{e q}$ or $\lambda-\Gamma_{\infty}$ plane for aqueous SDS in mineral oil a)-c). The red + shows the equilibrium value found using the pattern search algorithm with three different initial conditions a)-c). 


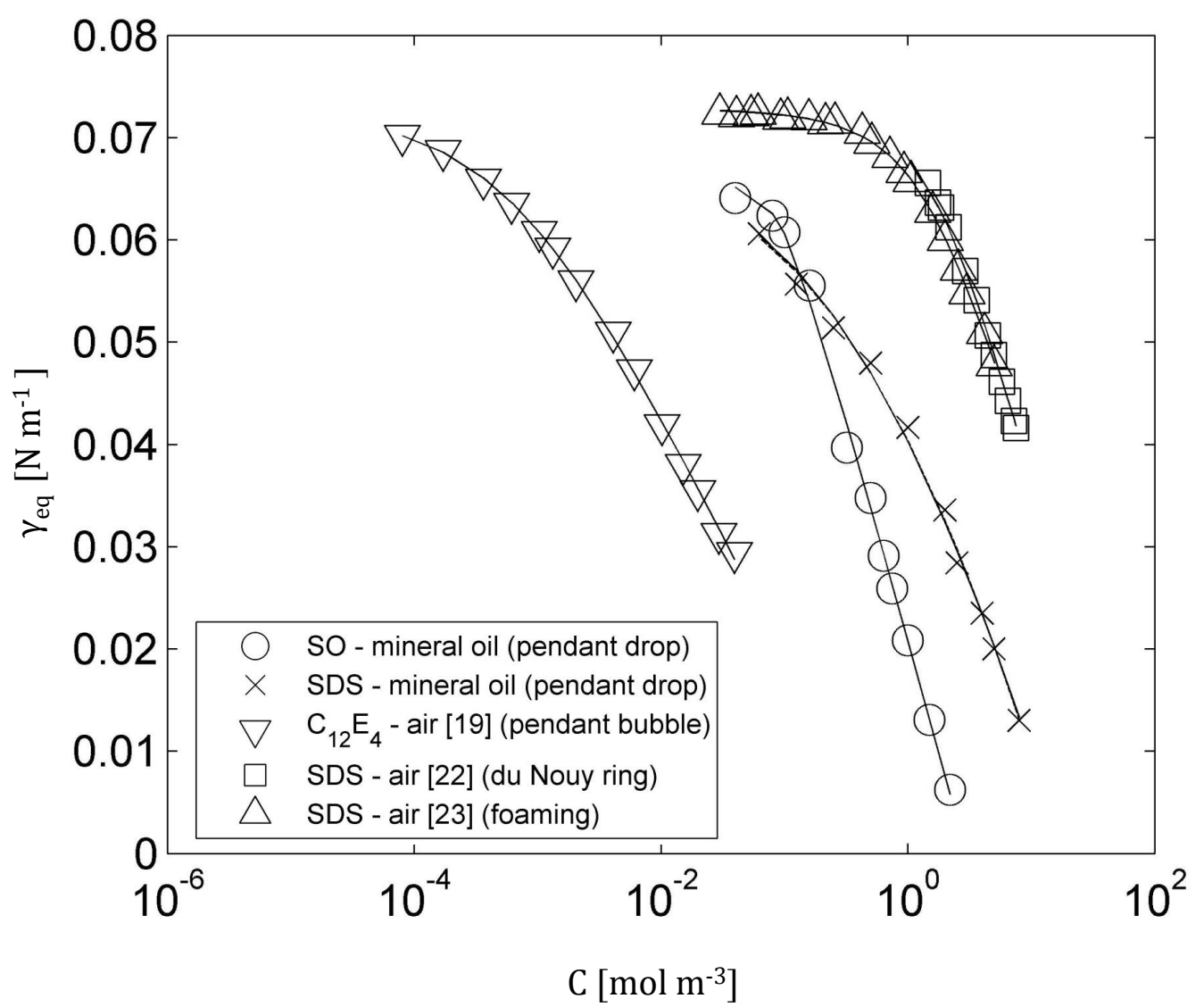

Figure 7: Plots of equilibrium surface tension versus bulk concentration for sodium oleate (SO) and sodium dodecyl sulfate (SDS). The lines are the best fit curve generated from the pattern search algorithm.

using the pattern search method. The sets include two pendant drops and one pendant bubble study: aqueous sodium oleate (SO) drops in mineral oil, aqueous sodium dodecyl sulfate (SDS) drops in mineral oil, and air bubbles in aqueous $C_{12} E_{4}$ [19]. We also include two historical studies involving aqueous SDS in air $[20,21]$ where equilibrium surface tension values are determined by foaming [22] and du Noüy ring [23] methods. The authors of this paper have produced the data for the two liquid-liquid pendant drop surface tension measurements. 
The objective function for the three dimensional isotherm curve fits are shown in each of three planes $\lambda-K_{e q}, \Gamma_{\infty}-K_{e q}$ and $\lambda-\Gamma_{\infty}$ in Figs. 5-6 in the vicinity of the local minimum. Fig. 5(a)-(c) shows data for (a) aqueous SO in mineral oil (pendant drop), (b) aqueous SDS in air [23] (du Noüy ring), and aqueous $\mathrm{C}_{12} \mathrm{E}_{4}$ in air [19] (pendant bubble). The red cross in each plot denotes the location of the minimum determined from the pattern search algorithm. Each row corresponds to the computation of the objective function for a given system. In each plot there is a clear local minimum as defined by the closed circles that surround the red cross in each graph. Fig. 6 shows data for the aqueous SDS in mineral oil (pendant drop) system. The initial conditions $\left(K_{e q, 0}, \Gamma_{\infty, 0}, \lambda_{0}\right)$ are varied to produce the three plots as indicated in the caption. Unlike the previous set of plots these do not necessarily yield closed domains in the first and last columns of data. They do appear to close if the graphs were continued in the positive $\lambda$ direction but this is difficult to say with certainty since the graphs evolve in three-dimensions.

The results of the pattern search minimization procedure are shown in Fig. 7 through plots of $\gamma$ versus $C$ for each system. The bulk surfactant concentrations span $10^{-4}$ to $10 \mathrm{mM}$ when including all the data sets. The individual data sets span a much smaller range of about two orders of magnitude for each system. The $\mathrm{C}_{12} \mathrm{E}_{4}$ non-ionic surfactant data spans the lowest concentration values while the SDS surfactant systems span the largest. The surface tension values range from a minimum less than $10 \mathrm{mN} \mathrm{m}^{-1}$ for the aqueous $\mathrm{SO}$ in mineral oil to values that are near the clean interface case for water in air $72 \mathrm{mN} \mathrm{m}^{-1}$ and water in mineral oil $67 \mathrm{mN} \mathrm{m}^{-1}$. For the SDS in mineral oil data the minimization values for each of 
the three different initial conditions is plotted on the same figure where the lines overlap.

\section{Discussion}

The pattern search method appears to be a robust and efficient means of finding the best fit of eqs. 4-6 for a given experimental drop shape. The pattern search algorithm was capable of fitting pendant drop and bubble halves with surface tensions up to $72.39 \mathrm{mN} \mathrm{m}^{-1}$ and as low as $0.54 \mathrm{mN} \mathrm{m}^{-1}$ using the same initial guess for $\gamma$ as shown in Fig. 3. The drop and bubble in Fig. 4(b) and 4(c) have lower $B o^{*}$ and this typically means the pendant drop method risks returning less accurate results [4]. The corresponding contour plots of $\psi_{R}$ in Fig. 3(b) and 3(c) provide a visual explanation. When compared to the drop in Fig. 4(a), the level curves of $\psi_{R}$ become elongated in the $\gamma$-direction as you approach the best fit. Given that the same convergence criteria is used in Fig. 3(a)-(c), this means that a wider range of $\gamma$ could satisfy those criteria in Fig. 3(b) and 3(c). This effect would become magnified as the drop shape approaches a sphere, leading to a wider range $\gamma$ potentially satisfying the convergence criteria. Then in order to accurately fit a low $B o^{*}$ drop, one would need a sufficient combination of high image resolution and strict convergence criteria.

Using $\gamma_{0}=5 \Delta \rho g b_{x}^{2}$, the four example fits of $I_{R}$ in Fig. 3 were determined within 0.95 and $1.54 \mathrm{~s}$ for pendant drops and bubbles with widely varying sizes and $\gamma$. Further analysis of the computation time is provided in Fig. 8 where transient surface tension measurements of 


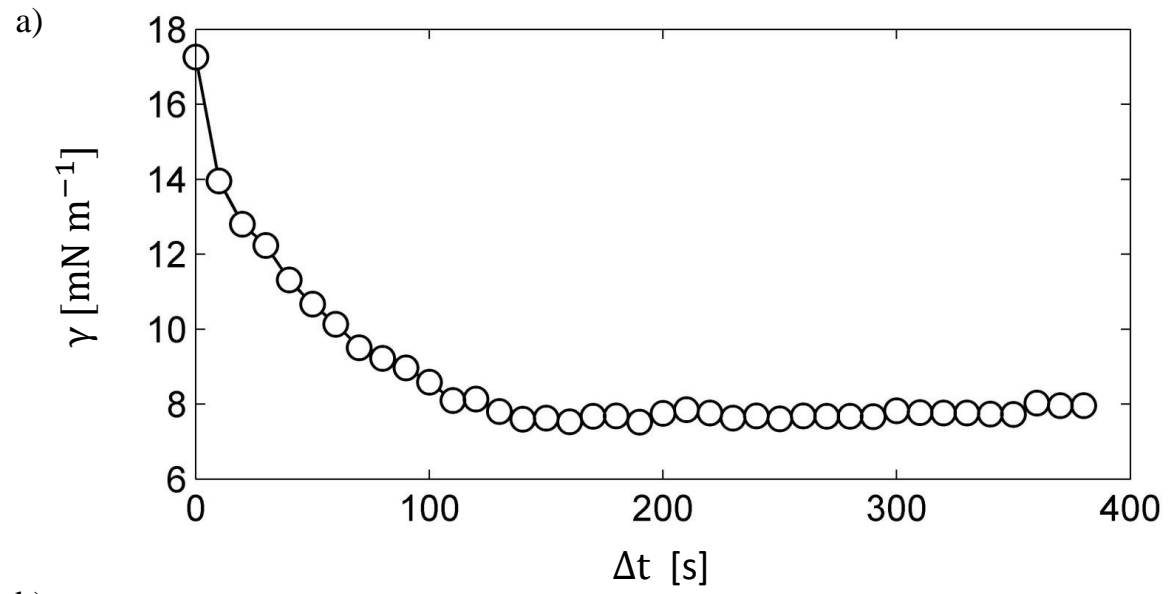

b)

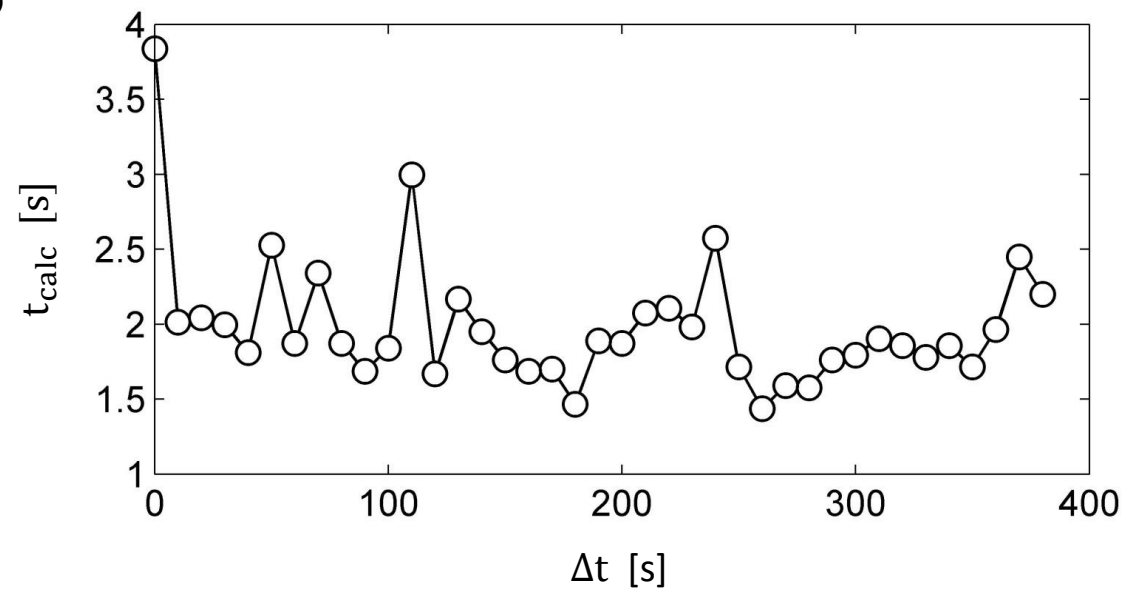

Figure 8: a plot of (a) $\gamma(t)$ for a drop of $2 \mathrm{mM}$ SO in mineral oil is shown along with (b) the total time taken to fit eqs. 4-6 to both $I_{L}$ and $I_{R}$ for each experimental image. 
a 2 mM SO drop in mineral oil are plotted. In Fig. 8(a) $\gamma(t)$ is shown representing successive fits to experimental pendant drop images taken every $10 \mathrm{~s}$ for just over 6 min. Figure 8(b) then shows the time taken to fit both $I_{L}$ and $I_{R}$ for each image, $t_{c a l c}$. For each image $I_{L}$ and $I_{R}$ had roughly 630 coordinates. The $B o^{*}$ for the first image in Fig. $8(\mathrm{a})$ is 0.073 and at the last image $B o^{*}=0.15$. The first image took nearly $4 \mathrm{~s}$ to fit, but subsequent images took roughly half the time since initial guesses for $b_{0}$ and $\gamma_{0}$ were taken as the solution of the previous time step. The total time taken to fit the 39 images in Fig. 8 is $77.3 \mathrm{~s}$ or 1.98 s per image. This is significantly faster than the time interval between successive images. Thus the pattern search algorithm can be used to fit experimental images while the images are being captured simultaneously. This would be true for many pendant drop experiments save for ones requiring very short time intervals between images.

A summary of the estimates for the surfactant transport parameters determined by fitting the equilibrium surface tension data to the three parameter Frumkin isotherm are presented in Table 1. The first column show the estimates for $\Gamma_{\infty}$ where all values are similar and range from $3.47-4.22 \times 10^{-6}$. The SDS in air [23] has the lowest value while the non-ionic $\mathrm{C}_{12} \mathrm{E}_{4}$ has the largest. The trends are similar for the estimates for the bulk surfactant distribution coefficient $K_{e q}$ which span some 4 orders of magnitude for the range of data presented.

Perhaps the most interesting results from the estimates of the surfactant transport parameters appear in the last two columns which provide information for the molecular interaction parameter. This parameter has been the subject of much debate where it is now generally 


\begin{tabular}{lcccc}
\hline & $\Gamma_{\infty}$ & $K_{e q}$ & $\lambda$ & $\lambda_{\text {crit }}$ \\
& $\mathrm{mol} \mathrm{m}^{-2}$ & $\mathrm{~m}^{3} \mathrm{~mol}^{-1}$ & - & - \\
\hline $\mathrm{SO}$ - mineral oil (pendant drop) & $3.89 \times 10^{-6}$ & 2.16 & 3.21 & 1.89 \\
$\mathrm{SDS}$ - mineral oil (pendant drop) & $3.84 \times 10^{-6}$ & 10.6 & -3.88 & 21.7 \\
$\mathrm{C}_{12} \mathrm{E}_{4}$ - air [19] (pendant bubble) & $4.22 \times 10^{-6}$ & 2615 & -1.07 & 33.18 \\
$\mathrm{SDS}$ - air [22] (foaming) & $3.86 \times 10^{-6}$ & 0.214 & 2.02 & 1.07 \\
$\mathrm{SDS}$ - air [23] (du Noüy ring) & $3.47 \times 10^{-6}$ & 0.356 & 1.45 & 1.30 \\
\hline
\end{tabular}

Table 1: Frumkin isotherm model constants

understood that there is an upper limit of $\lambda=4$ above which the isotherm provides multiple solutions. Here we have proposed another critical $\lambda$ based on the $C_{\infty}[24]$ and $K_{e q}$ which may be initiated using data provided from the Langmuir isotherm. For $\lambda>\lambda_{\text {crit }}$ there is only one unique solution to Eq. 10 used to determine stability. This hypothesis is confirmed in the set of data presented in Table 1. Therefore, any negative $\lambda$ should be viewed with suspicion given this criteria. In this regard the equilibrium surface tension data for the SDSmineral oil does produce multiple solutions where we have chosen the smallest value because it provides the smallest $\Gamma_{\infty}$ (largest ratio of $\Gamma_{e q} / \Gamma_{\infty}$ ). The $\mathrm{C}_{12} \mathrm{E}_{4}$ also produces a negative $\lambda$ but is a special case because the discriminant in Eq. 10 is extremely small such that it is essentially the same as having one unique non-trivial solution i.e. it is conditionally stable. This is somewhat confirmed in the plots shown in Fig. 5 where the objective functions for $\mathrm{C}_{12} \mathrm{E}_{4}$ has a very narrow band in both planes containing $\lambda$. So for systems with large $K_{e q}$ it is possible to find a unique solution through minimization. 


\section{Conclusions}

Surface tension measurements were made using axisymmetric drop shape analysis (ADSA) of a pendant drop with the pattern search method as the minimization algorithm. Here, it has been successfully used to fit ADSA data to numerical solutions of Young-Laplace equation without the need to calculate gradients. This is in contrast to the otherwise nearly universal use of gradient based minimization methods [1]. The non-gradient based NelderMead simplex method [2] has been used in the context of the pendant drop method by using the MATLAB function fminsearch [4], but the pattern search method used here has been mathematically proven to be capable of consistently converging to a local minimum $[5,6]$.

After producing equilibrium surface tension data from pendant drops for aqueous sodium oleate (SO) and sodium dodecyl sulfate (SDS) in mineral oil we fit this data to the Frumkin isotherm also using the pattern search method. We analyze the convergence criteria for the equation and extend the upper bound limit for the molecular interaction parameter $\lambda$ using a Taylor series expansion of the Frumkin isotherm equation for small fractional surface cov-

erage. The curve fits from the pattern search method and stability criteria determined from linearization of the Frumkin isotherm fit the data fairly well.

Future analysis of the pattern search method for ADSA should include sessile drops. It may 
also be possible to apply the method for direct estimates of adsorption/desorption rates in single and multi-surfactant systems using transient surface tension data.

\section{Acknowledgements}

This work supported by NSF (CBET \#1262718).

\section{References}

[1] Y. Rotenberg, L. Boruvka, A.W. Neumann, Determination of surface tension and contact angle from the shapes of axisymmetric fluid interfaces. J. Colloid Interface Sci. 93 (1983) 169-183.

[2] J.A. Nelder, R. Mead, A simplex method for function minimization. Comput. J. 7 (1965) 308-313.

[3] T.G. Kolda, R.M. Lewis, V. Torczon, Optimization by direct search: New perspectives on some classical and modern methods. SIAM Rev. 45 (2003) 385-482.

[4] N.J. Alvarez, L.M. Walker, S.L. Anna, A non-gradient based algorithm for the determination of surface tension from a pendant drop: Application to low Bond number drop shapes. J. Colloid Interface Sci. 333 (2009) 557-562.

[5] W. Yu, Positive basis and a class of direct search techniques, Scientia Sinica, Special Issue of Mathematics 1 (1979) 5367. 
[6] V. Torczon, On the convergence of pattern search algorithms. SIAM J. Optim. 7 (1997) $1-25$.

[7] A. Kalantarian, S.M.I. Saad, A.W. Neumann, Accuracy of surface tension measurement from drop shapes: the role of image analysis. Adv. Colloid Interface Sci. 199 (2013) 1522.

[8] N. Otsu, A threshold selection method from gray-level histograms. IEEE 1 (1979) 62-66.

[9] C. Huh, R.L. Reed, A method for estimating interfacial tensions and contact angles from sessile and pendant drop shapes. J. Colloid Interface Sci. 91 (1983) 472-484.

[10] F. Bashforth, J.C. Adams, An attempt to test the theories of capillary action. Cambridge University Press, london, 1883.

[11] S.Y. Lin, K. McKeigue, C. Maldarelli, Diffusion-controlled surfactant adsorption studied by pendant drop digitization. AIChE J. 36 (1990) 1785-1795.

[12] W.M. Haynes, T.J. Bruno, D.R. Lide, CRC Handbook of Chemistry and Physics, 95th ed., CRC Press, Boca Raton, 2015.

[13] B. von Szyszkowski, Experimental studies of the capillary porperties of aqueous solutions of fatty acids. B. Z. Phys. Chem. 64 (1908) 385-414.

[14] I. Langmuir, The constitution and fundamental properties of solids and liquids. II. Liquids. J. Am. Chem. Soc. 39 (1917) 1848-1905. 
[15] C.-H. Chang, E.I. Frances, Adsorption dynamics of surfactants at the air/water interface: a critical review of mathematical models, data, and mechanisms. Colloids Surf., A: Physicochem. Eng. Aspects 100 (1995) 1-45.

[16] E.H. Lucassen-Reynders, Surface equation of state for ionized surfactants. J. Phys. Chem. 70 (1965) 1777-1785.

[17] A.J. Prosser, E.I. Franses, Adsorption and surface tension of ionic surfactants at the air-water interface: review and evaluation of equilibrium models. Colloids Surf., A: Physicochem. Eng. Aspects 178 (2001) 1-40.

[18] J. Eastoe, S. Nave, A. Downer, A. Paul, A. Rankin, K. Tribe, Adsoprtion of ionic surfactants at the air-solution interface. Langmuir 16 (2000) 4511-4518.

[19] C.-T. Hsu, M.-J. Shao, S.-Y. Lin, Adsorption kinetics of $\mathrm{C}_{12} \mathrm{E}_{4}$ at the air-water interface: adsorption onto a fresh surface. Langmuir 16 (2000) 3187-3194.

[20] K. Lunkenheimer, G. Czichocki, R. Hirte, W. Barzyk, Novel results on the adsorption of ionic surfactants at the air/water interface - sodium- $n$-alkyl sulphates. Colloids Surf., A: Physicochem. Eng. Aspects 101 (1995) 187-197.

[21] V.B. Fainerman, S.V. Lylyk, E.V. Akensenko, J.T. Petkov, J. Yorke, R. Miller, Surface tension isotherms, adsorption dynamics and dilational visco-elasticity of sodium dodecyl sulphate solutions. Colloids Surf., A: Physicochem. Eng. Aspects 354 (2010) 8-15.

[22] K.J. Mysels, Surface tension of solutions of pure sodium dodecyl sulfate. Langmuir 2 (1986) 423-428. 
[23] P. Warszyński, W. Barzyk, K. Lunkenheimer, H. Fruhner, Surface tension and surface potential of Na $n$-Dodecyl Sulfate at the air-solution interface: Model and experiment. J. Phys. Chem. B 102 (1998) 10948-10957.

[24] Critical Micelle Concentrations of Aqueous Surfactant Systems, U.S. Department of Commerce, U. S. Government Printing Office: Washington D.C. 1971. 


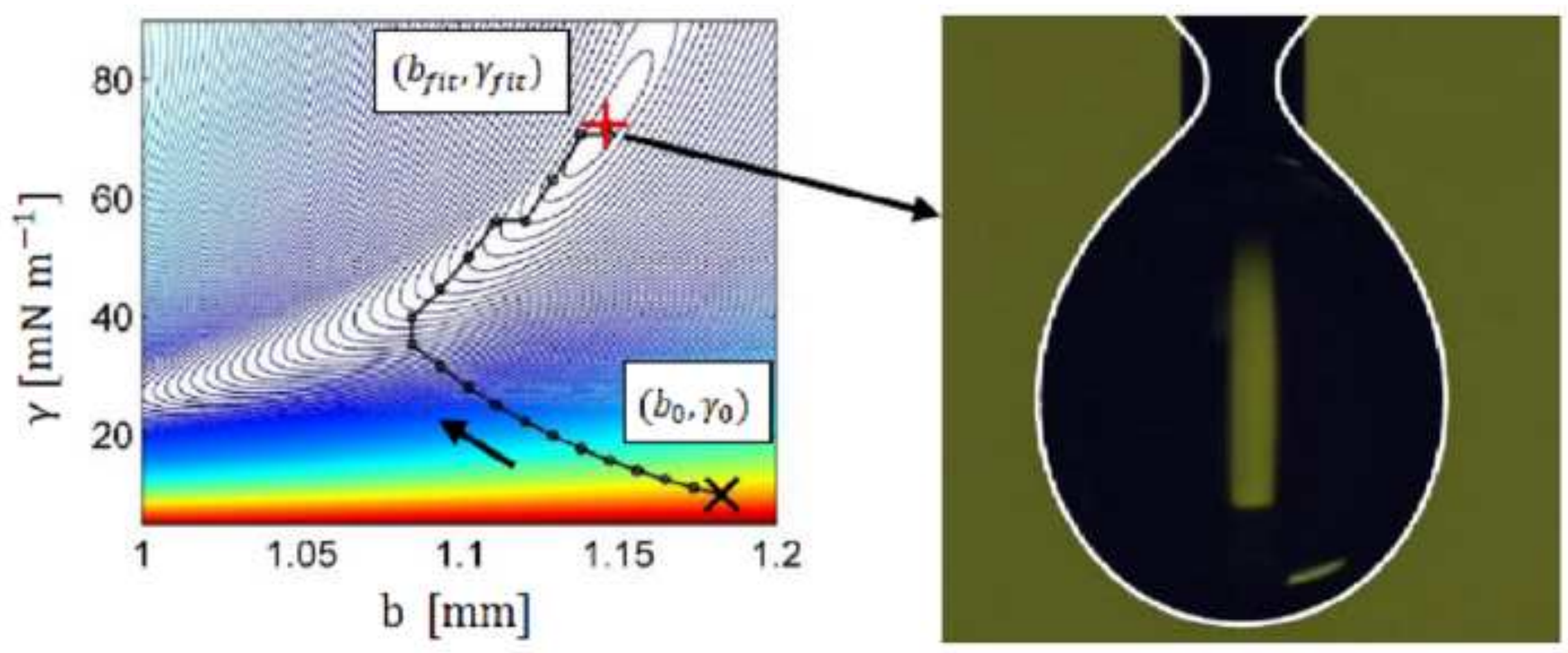

